\title{
A NOTE ON LIPSCHITZ CLASSES
}

\author{
BY E. S. QUADE
}

This note consists in the application of some results of Hardy and Littlewood* on fractional integrals to a theorem of Paley and Zygmund $\dagger$ and gives a generalization of that theorem.

We consider only functions of the Fourier power series type. That is, $f(x)$ is periodic in $2 \pi$, integrable, and with a Fourier series of the form

$$
f(x) \backsim \sum_{n=0}^{\infty} c_{n} e^{i n x}, \quad c_{n}=\frac{1}{2 \pi} \int_{-\pi}^{\pi} f(x) e^{-i n x} d x .
$$

In dealing with functions of the class $\operatorname{Lip}(\alpha)$ or $\operatorname{Lip}(\alpha, p), \alpha \neq 1$, this restriction is a matter of convenience rather than one of necessity. $\ddagger$

A function $f(x)$ is said to belong to the class $\operatorname{Lip}(\alpha)$, where $0 \leqq \alpha \leqq 1$, in the interval $(-\pi, \pi)$, if

$$
f(x+h)-f(x-h)=O\left(h^{\alpha}\right)
$$

uniformly for $-\pi \leqq x-h<x+h \leqq \pi$, and to $\operatorname{Lip}(\alpha, p)$, where $p \geqq 1,0 \leqq \alpha \leqq 1$, in $(-\pi, \pi)$, if $f(x) \epsilon L$, and

$$
\int_{-\pi}^{\pi}|f(x+h)-f(x-h)|^{p} d x=O\left(h^{\alpha p}\right) .
$$

The functions $\phi_{n}(t),(n=0,1,2, \cdots)$, are the Rademacher functions. $\$$

* Hardy and Littlewood, Some properties of fractional integrals I, Mathematische Zeitschrift, vol. 27 (1927-28), pp. 565-606. We will refer to this paper as (HL).

$\dagger$ Paley and Zygmund, On some series of functions, Proceedings Cambridge Philosophical Society, vol. 26 (1930), pp. 337-357. A. Zygmund, Trigonometrical Series, $1935, \S 5.61$. We will refer to this book as (Z). It contains extensive bibliographical references.

$\ddagger$ Hardy and Littlewood, $A$ convergence criterion for Fourier series, Mathematische Zeitschrift, vol. 28 (1928), pp. 612-634, in particular, $\$ 2$ and $\$ 3.5$. See also $(Z), \S 7.4$.

$\S$ For definitions and properties see $(Z), \S \S 1.32$ and 5.5 to 5.61 . 
THEOREM 1. Let $c_{0}, c_{1}, c_{2}, \cdots, c_{n}, \cdots$ be a sequence of real or complex numbers such that

$$
\sum_{n=2}^{\infty} n^{2 \alpha}\left|c_{n}\right|^{2}(\log n)^{1+\epsilon}
$$

converges for an $\epsilon>0$. Then, for almost all values of $t$, the function

$$
f_{t}(x) \backsim \sum_{n=0}^{\infty} c_{n} e^{i n x} \phi_{n}(t)
$$

belongs to the class $\operatorname{Lip}(\alpha),(0 \leqq \alpha \leqq 1)$. The theorem is false for the case $\alpha=0$ or $\alpha=1$ if $\epsilon=0$.

As a consequence of the theorem of Paley and Zygmund mentioned above it follows that

$$
f_{t}^{\alpha}(x)=i^{\alpha} \sum_{n=1}^{\infty} n^{\alpha} c_{n} e^{i n x} \phi_{n}(t),
$$

for almost all values of $t$, is a continuous function (since the series converges uniformly we put $f_{t}^{\alpha}(x)$ equal to the sum of the series). That is, we have

$$
f_{t}^{\alpha}(x) \epsilon \operatorname{Lip}(0) .
$$

If by the symbol $f_{t, \alpha}^{\alpha}(x)$ we denote the integral of $f_{t}^{\alpha}(x)$ of order $\alpha$, we have*

$$
f_{t, \alpha}^{\alpha}(x) \epsilon \operatorname{Lip}(\alpha)
$$

But

$$
\begin{aligned}
f_{t, \alpha}^{\alpha}(x) & =i^{\alpha} \sum_{n=1}^{\infty} \frac{c_{n} n^{\alpha}}{(i n)^{\alpha}} e^{i n x} \phi_{n}(t) \\
& =f_{t}(x)-c_{0} \phi_{0}(t) .
\end{aligned}
$$

To show that the theorem is not true in the case $\alpha=1$, for $\epsilon=0$, we consider the function

* (Z), $\$ \S 9.80$ and 9.81. A function satisfies a condition $\operatorname{Lip}^{*}(\alpha)$ or $\operatorname{Lip}^{*}(\alpha, p)$ when it satisfies a condition analogous to that for $\operatorname{Lip}(\alpha)$ or $\operatorname{Lip}(\alpha, p)$ but with $o$ small in place of $O$ large. In each of our theorems $\operatorname{Lip}(\alpha)$ or $\operatorname{Lip}(\alpha, p)$ may be replaced by $\operatorname{Lip}^{*}(\alpha)$ or $\operatorname{Lip}^{*}(\alpha, p)$, respectively, except in the case $\alpha=1$; this follows from Theorems 18, 21, and 22 of (HL). 


$$
\sum_{m=1}^{\infty} \frac{ \pm i e^{i 2^{m} x}}{2^{m} m \log (m+1)}
$$

This can not belong to $\operatorname{Lip}(1)$ for any sequence of signs since it is the integral of

$$
\sum_{m=1}^{\infty} \frac{ \pm e^{i 2^{m} x}}{m \log (m+1)}
$$

which is Paley and Zygmund's example* of a series which does not represent a bounded function for any sequence of signs.

For the case of $\operatorname{Lip}(\alpha, p),(0 \leqq \alpha \leqq 1, p \geqq 1)$, we have a similar theorem.

Theorem 2. Let $c_{0}, c_{1}, c_{2}, \cdots, c_{n}, \cdots$ be a sequence of real or complex numbers such that $\sum_{n=1}^{\infty} n^{2 \alpha}\left|c_{n}\right|^{2}$ converges. Then, for almost all values of $t$, the function

$$
f_{t}(x) \backsim \sum_{n=0}^{\infty} c_{n} e^{i n x} \phi_{n}(t)
$$

belongs to the class $\operatorname{Lip}(\alpha, p),(p \geqq 1,0 \leqq \alpha \leqq 1)$.

Since $\sum_{n=1}^{\infty}\left|n^{\alpha} c_{n}\right|^{2}$ is convergent, it follows that $\dagger$

$$
f_{t}^{\alpha}(x) \backsim i^{\alpha} \sum_{n=1}^{\infty} n^{\alpha} c_{n} e^{i n x} \phi_{n}(t)
$$

belongs to $L_{p},(p \geqq 1)$.

Now by a theorem of Hardy and Littlewood $\ddagger$

$$
f_{t, \alpha}^{\alpha}(x) \epsilon \operatorname{Lip}(\alpha, p)
$$

But, as before,

$$
f_{t, \alpha}^{\alpha}(x)=f_{t}(x)-c_{0} \phi_{0}(t)
$$

As a corollary of the following theorem we have a better theorem for the case $1 \leqq p \leqq 2$.

* Paley and Zygmund, loc. cit., p. 350. This gives the case $\alpha=0, \epsilon=0$.

$\dagger(Z), \$ 5.6$ (iii).

$\ddagger(\mathrm{HL})$, Theorems 21, 22, and ff. 
TheOREM 3. If

$$
\sum_{n=-\infty}^{+\infty} n^{p^{\prime} \alpha}\left|c_{n}\right|^{p^{\prime}}, \quad\left(1<p^{\prime} \leqq 2\right)
$$

converges, then

$$
f(x) \backsim \sum_{n=-\infty}^{+\infty} c_{n} e^{i n \alpha}
$$

belongs to the class $\operatorname{Lip}(\alpha, p), 1 / p+1 / p^{\prime}=1,(0 \leqq \alpha \leqq 1)$.

From the Young-Hausdorff* theorem we have

$$
\left(\frac{1}{2 \pi} \int_{-\pi}^{\pi}\left|f^{\alpha}(x)\right|^{p} d x\right)^{1 / p} \leqq\left(\sum_{n=-\infty}^{+\infty}\left|n^{\alpha} c_{n}\right|^{p^{\prime}}\right)^{1 / p^{\prime}},
$$

where

$$
f^{\alpha}(x) \backsim i^{\alpha} \sum_{n=-\infty}^{+\infty} n^{\alpha} C_{n} e^{i n x} .
$$

Since $f^{\alpha}(x) \epsilon L_{p}$, we have $\dagger$

$$
f(x)-c_{0}=f_{\alpha}^{\alpha}(x)=\sum_{n=-\infty}^{+\infty} c_{n} e^{i n x} \epsilon \operatorname{Lip}(\alpha, p), \quad(p \geqq 2) .
$$

Corollary. If

$$
\sum_{n=-\infty}^{+\infty} n^{2 \alpha}\left|c_{n}\right|^{2}, \quad(0 \leqq \alpha \leqq 1),
$$

converges, then

$$
f(x) \backsim \sum_{n=-\infty}^{+\infty} c_{n} e^{i n x}
$$

belongs to Lip $(\alpha, p)$ for every $p$ such that $1 \leqq p \leqq 2$.

This follows because

$$
\left(\frac{1}{2 \pi} \int_{-\pi}^{\pi}|f(x)|^{p} d x\right)^{1 / p} \leqq\left(\frac{1}{2 \pi} \int_{-\pi}^{\pi}|f(x)|^{2} d x\right)^{1 / 2} .
$$

\section{BROWN UNIVERSITY}

* (Z), \$9.1.

$\dagger(\mathrm{HL})$, Theorem 21. 